\title{
Maximising Opportunities Post Coronavirus Disease 2019: Time to Embrace a New Era of Atrial Fibrillation Research
}

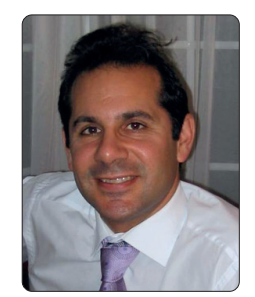

Disclosure: PDL is supported by UCL/UCLH Biomedicine NIHR and Barts BRC and discloses research grants and speaker fees from Boston Scientific, Abbott and Medtronic.

Citation: Arrhythmia \& Electrophysiology Review 2021;10(2):63-4. DOI: https://doi.org/10.15420/aer.2021.29

Correspondence: Pier D Lambiase, Barts Heart Centre, West Smithfield, London EC1A 7BE, UK. E: p.lambiase@ucl.ac.uk

Open Access: This work is open access under the CC-BY-NC 4.0 License which allows users to copy, redistribute and make derivative works for non-commercial purposes, provided the original work is cited correctly.

$\mathrm{n}$ this edition of Arrhythmia \& Electrophysiology Review there is a strong emphasis on different aspects of AF care, including the use of remote monitoring accelerated by the coronavirus disease 2019 (COVID-19) pandemic, stroke risk and optimisation of therapy with ablation. Indeed, an elegant mechanistic paper highlights the random nature of AF proposing a novel concept of event analysis.'

However, a fundamental issue in current AF management still revolves around the lack of adequately powered multicentre trials that address key questions pertaining to making real differences to patient outcomes and quality of life. This is not surprising, as high quality randomised controlled trials are expensive along with being extremely difficult to design and execute. These challenges ultimately result in smaller scale technologydriven studies assessing the efficacy of new ablation technologies. Such approaches are manageable in terms of recruitment and have easily tractable outcomes, such as proof of pulmonary isolation on repeat electrophysiology study or reduction in overall AF burden.

CABANA is the most ambitious AF trial to date. However, to trial purists it ultimately remains inconclusive as endpoints were changed during the study and the per assigned protocol analysis failed to show benefit of ablation over medical therapy for the combined composite endpoint. ${ }^{2}$ About halfway into the CABANA trial, the death/stroke/bleeding/cardiac arrest composite secondary endpoint was elevated to become the primary endpoint when it became clear that the initial enrolment target of 3,000 patients would not be achieved and the number of deaths would not be large enough to draw reliable conclusions. The original primary endpoint - all-cause mortality - became a secondary endpoint. The resulting patient treatment received data were positive but the trial suffers from the risk of bias, leading to on-going debate in the field.

CASTLE AF yielded highly positive results for the efficacy of AF ablation in heart failure, reducing mortality and hospitalisations, but this has not lead to a dramatic increase in clinical uptake. ${ }^{3,4}$ This is mainly because there remains considerable scepticism among physicians in the heart failure community since the majority of heart failure patients they see would not have been eligible for the trial, which focused on patients with implanted devices who failed anti-arrhythmic therapy. Even recruitment involved an 8:1 screening to enrolment ratio. Similarly, the RAFT-AF trial recently reported at the 2021 American College of Cardiology meeting failed to reach its primary endpoint of mortality and heart failure hospitalisation comparing ablation to rate control, mainly because it was underpowered to show a significant difference. ${ }^{5}$

This leaves the field at a crossroads in progressing ablation therapies for AF in a meaningful direction for patients and to convince health economists and governments regarding the utility of ablation - especially in persistent AF. This must ultimately be determined by patient selection and trial design with adequate sample sizes to detect meaningful differences in quality of life, hospitalisations and mortality. Furthermore, the concept of 30 seconds of AF representing a failure of therapy is a very high bar to reach and raises the question as to whether reduction in AF burden is a more realistic endpoint to evaluate. ${ }^{6}$

Until now the majority of AF research has focused on technological developments and novel mapping strategies to identify 'rotors' or other sites of organised activity with positive results that have not translated at scale. This is most likely because of a combination of initial positive reporting bias and very careful case selection in single-centre studies.

In order to solve these challenges, wider clinical trial enrolment and follow-up requires the implementation of more electronic-data-driven strategies to collect outcomes at scale and draw meaningful conclusions for patients. In many ways, the pandemic has opened up the pathways to achieve this, with the necessary embracement of remote monitoring and electronic data collection now routinely employed in clinical care. With the availability of linked electronic health records in some countries, such as the UK through the NHS, one can track all hospital and medical encounters. Coupled with wearable devices, mobile phone apps, pacemakers/ICDs and loop recorders, one has the tools to truly track outcomes of interventions at scale and recruit real-world patients in interventional studies This revolution in the mindset of clinicians should provide the foundation for much smarter trial designs recruiting patients at scale and digital data capture with minimal human effort. 
Indeed, this infrastructure provides the opportunity to undertake cluster randomisation studies at scale where patients can be allocated one treatment in one geographical/hospital group and an alternative in another, switching after 6 months to 1 year. ${ }^{8}$ This would enable recruitment of patients at scale and give statistically robust data. Such an approach has been employed in primary care to optimise AF management. ${ }^{9}$ Similarly, adaptive trial designs would mean that once one arm of a trial demonstrates futility, the arm could be changed accordingly whilst continuing to recruit new patients. This high throughput study design strategy was employed extremely effectively in the RECOVERY trial during the pandemic, discovering the utility of dexamethasone and proving the non-efficacy of chloroquine, lopinavir-ritonavir and colchicine. ${ }^{10}$ Such trial designs would lend themselves easily to specific drug or ablation strategies in AF.

Therefore, the revolution in using remote data for clinical care and wearable technologies necessitated by the pandemic has given rise to new opportunities for data-driven research strategies and trial design. This should lead the way to execute clinical trials at scale with data collection integrated into usual care to provide a robust evidence base for AF treatment in the next decade.

\section{Pier D Lambiase}

Section Editor, Arrhythmia Risk Stratification, Arrhythmia \& Electrophysiology Review

Institute of Cardiovascular Science, University College London, London, UK; Barts Heart Centre, West Smithfield, London, UK

1. Quah JX, Dharmaprani D, Lahiri A, et al. Reconceptualising atrial fibrillation using renewal theory: a novel approach to the assessment of atrial fibrillation dynamics. Arrhythm Electrophysiol Rev 2021;10:78-85. https://doi.org/10.15420/aer.2020.42.

2. Packer DL, Mark DB, Robb RA, et al. Effect of cathete ablation vs antiarrhythmic drug therapy on mortality, stroke, bleeding, and cardiac arrest among patients with atrial fibrillation: the CABANA randomized clinical trial. JAMA 2019;321:1261-74. https://doi.org/10.1001/ jama.2019.0693; PMID: 30874766

3. Marrouche NF, Brachmann J, Andresen D, et al. Catheter ablation for atrial fibrillation with heart failure. N Engl J Med 2018;378:417-27. https://doi.org/10.1056 NEJMoa1707855; PMID: 29385358

4. Noseworthy PA, Van Houten HK, Gersh BJ, et al.
Generalizability of the CASTLE-AF trial: catheter ablation for patients with atrial fibrillation and heart failure in routine practice. Heart Rhythm 2020;17:1057-65. https://doi.org/10.1016/j.hrthm.2020.02.030; PMID: 32145348.

5. American College of Cardiology. RAFT-AF: Rhythm and rate control similar for death, HF progression in patients with AFib and HF. 17 May 2021. https://www.acc.org/ latest-in-cardiology/articles/2021/05/12/19/40/mon1045am-raft-af-acc-2021 (accessed 14 June 2021).

6. Wechselberger S, Kronborg M, Huo Y, et al. Continuous monitoring after atrial fibrillation ablation: the LINQ AF study. Europace 2018;20:f312-20. https://doi.org/10.1093/ europace/euy038; PMID: 29688326.

7. van den Dries CJ, van Doorn S, Rutten FH, et al. Integrated management of atrial fibrillation in primary care: results of the ALL-IN cluster randomized trial. Eur Heart J 2020;41:2836-44. https://doi.org/10.1093/ eurheartj/ehaa055; PMID: 32112556

8. Ajmera Y, Singhal S, Dwivedi SN, Dey AB. The changing perspective of clinical trial designs. Perspect Clin Res 2021;12:66-71. https://doi.org/10.4103/picr.PICR_138_20; PMID: 34012901

9. Guo Y, Guo J, Shi X, et al. Mobile health technologysupported atrial fibrillation screening and integrated care: a report from the mAFA-II trial Long-term Extension Cohort. Eur J Intern Med 2020;82:105-11. https://doi. org/10.1016/j.ejim.2020.09.024; PMID: 33067121.

10. University of Oxford. RECOVERY. Azithromycin results. Oxford: University of Oxford, 14 December 2020. https:// www.recoverytrial.net/results/azithromycin-results (accessed 14 June 2021). 